Research Article

\title{
Integrated Fault Detection and Fault Tolerant Control for Switched Systems with Asynchronous Switching
}

\author{
Supeng Zhu, ${ }^{1}$ Haoyu Cheng ${ }^{(D},{ }^{1}$ Wenxing Fu, ${ }^{2}$ Xiaohan Zhao, ${ }^{1}$ Wenyuan $\mathrm{Li}^{1},{ }^{1}$ and \\ Yifeng $\mathrm{Ma}^{1}$ \\ ${ }^{1}$ School of Astronautics, Northwestern Polytechnical University, Xi'an 710072, China \\ ${ }^{2}$ Unmanned System Research Institute, Northwestern Polytechnical University, Xi'an 710072, China
}

Correspondence should be addressed to Haoyu Cheng; chenghaoyu@nwpu.edu.cn

Received 25 January 2020; Revised 11 May 2020; Accepted 25 May 2020; Published 17 August 2020

Academic Editor: Libor Pekař

Copyright (C) 2020 Supeng Zhu et al. This is an open access article distributed under the Creative Commons Attribution License, which permits unrestricted use, distribution, and reproduction in any medium, provided the original work is properly cited.

The problem of integrated fault detection and fault tolerant control for switched systems with asynchronous switching is focused on in this paper. Based on the switched model, the inherent asynchronous switching is taken into consideration. The asynchronous switching means that the switching of filters/controllers will always lag behind the switching of modes, which will degrade the performance of the closed-loop system. The Lyapunov functional method and mode-dependent average dwell time method are combined for the analysis of the finite-time stability of the switched system. The properties of each subsystem are taken into consideration, which are less conservative. To achieve optimal performance, the filters and controllers are designed simultaneously. The parameters of filters and controllers are given in the form of linear matrix inequalities. In the end, the numerical example is given to illustrate the effectiveness of the proposed method.

\section{Introduction}

Switched system is a higher-level abstraction of hybrid system, which consists of a series of continuous-time (or discrete-time) subsystems and switching signal governing the switching among the subsystems $[1,2]$. Switched systems provide a unified framework for flexible modeling tool for many physical or manmade systems with switching feature, such as flight control systems [3], industrial electronics [4], and networked control systems $[5,6]$. Because of the advantages in modeling and controller design, great advancements have been obtained in both theory and application recently. Among these researches, the problems of stability and stabilization analysis $[7,8]$, controller design $[9,10]$, and filter design $[6]$ have been investigated. To mention a few, the problem of stability of switched systems is studied in [11], whose switching sequences are generated by a Muller automaton. The sliding mode controller for semi-Markovian jump systems via output feedback is designed in [12]. The sufficient condition on the sliding surface synthesis is presented and the stochastic stability of Markovian system is guaranteed. Moreover, the problem of output- feedback controller design for linear parameter-varying (LPV) system is addressed in [13]. The uncertainties of the scheduling parameters are taken into consideration and the stability and L2-gain performance of closed-loop system are guaranteed.

The basic problems of controller design are stability and stabilization analysis, which have been extensively studied in the past decades. The stability analysis methods in the Lyapunov sense mainly include common Lyapunov functional method and multiple Lyapunov functional method. It is well known that the common Lyapunov functional method is mainly applied in the problem of stability analysis of arbitrary switching. However, it is difficult to find a common Lyapunov function for all subsystems. Thus, it is necessary to consider stability of switched systems with constrained switching. It has been verified in existing researches that multiple Lyapunov functional method not only has flexibility but also can reduce the conservativeness. Moreover, in [14], the multiple Lyapunov functional method and average dwell time (ADT) method are combined to analyze the stability of switched system with asynchronous switching. The sufficient conditions to guarantee the stability 
of the closed-loop system are given in the form of linear matrix inequalities (LMIs). In addition, the information transformation relies on the network and there always exist time delay and data missing, which will cause the lag between the controllers and subsystems. Thus, there will exist matched periods and unmatched periods during each subsystem, which is called asynchronous switching. The energy of switched system will increase during the unmatched periods and decrease during the matched periods, which has arisen extensive attention in recent years. To decrease the conservativeness of ADT method, the modedependent average dwell time (MDADT) method is firstly introduced in [15]. The properties of each subsystem are taken into consideration, which leads to less conservative results. A new adaptive control method is proposed for a class of general switched uncertain nonlinear systems in [16]. An improved MDADT method is introduced to ensure the global boundedness of closed-loop system is guaranteed. The problem of adaptive tracking control for switched uncertain nonlinear systems with unmodeled dynamics is solved by adaptive control scheme. To deal with the problem of controller design for uncertain switched time-delay nonlinear systems with non-lower-triangular structure, the adaptive neural output-feedback controller is proposed in [17]. Neural networks, adaptive back-stepping technique, and variable separation method are applied to construct the controller. Lyapunov-Krasovskii functional method and ADT method are employed to guarantee the stability of closed-loop system. In [18], the problem of adaptive neural tracking control for uncertain switched nonlinear non-lower-triangular system is investigated. An adaptive neural tracking control method has been presented by using adaptive backstepping and multiple Lyapunov functions techniques, which can ensure that all signals remain bounded and the tracking error can eventually converge to a small neighborhood of the origin. Moreover, in [19], the problem of stability for slowly switched systems is investigated. The stability of switched system with stable subsystems and unstable subsystems is analyzed and the multiple discontinuous Lyapunov functional method is firstly derived. Tighter bounds on the dwell time are given and simulation results are provided to illustrate the advantages of the results.

With the increasing demands for higher reliability and high safety, the problems of fault detection and fault tolerant control attract more and more attention. The problem of fault detection filter design for nonlinear switched system is investigated in [20]. The persistent dwell time method and quasi-time-dependent Lyapunov functional method are combined to analyze the stability of the system. Moreover, a fuzzy-parameter-dependent fault detection filter is proposed to ensure the error system is stable with prescribed performance. A more general and less conservative method is introduced. The problem of robust passive fault detection for switched LPV systems with measurable and unmeasurable scheduling parameters is focused on in [21]. A switched Takagi-Sugeno (T-S) interval observer is proposed to estimate the state. Multiple Lyapunov functional method and ADT method are combined to analyze the stability of switched system. Sufficient conditions to ensure the convergence and the robustness of observer are given in the form of LMIs. In addition, to overcome the problem of interferences from actuator faults for partially unknown system, fault tolerant controller based on reinforcement learning is proposed [22]. Different from the traditional method, the fuzzy integral reinforcement learning fault tolerant tracking algorithm runs in real time, which is more reliable and sensitive to deal with the occurrence of failures. In [23], the observer-based fault estimation and active dynamic output feedback controller are designed for switched T-S fuzzy stochastic system. A novel piecewise fuzzy Lyapunov function is introduced and delay-dependent sufficient conditions to ensure the stability of closed-loop system are given in the form of LMIs. Finite-time boundedness is discussed and less conservative results are obtained. The reduced-order fault estimation observer is designed in [24], the dimension of the designed estimator is reduced, and the fault can be completely unknown and unbounded. The fault tolerant controller is proposed to stabilize the switched stochastic systems.

The results mentioned above are under the assumption that the fault detection filter and fault tolerant controller are designed separately. However, the performances of fault detection filters and fault tolerant controllers will influence each other. The output signal can be viewed as the input of fault detection filter, while the output of filter is the input of the controller. Thus, the traditional design method will no doubt give rise to conservativeness. In [25], the problem of closed-loop fault detection for flight vehicle is investigated. The flight vehicle is modeled as switched system and ADT method is applied to analyze the global stability of system. However, compared to MDADT method, ADT method will lead to conservative results. Moreover, most of the mentioned literature focus on the Lyapunov asymptotic stability, which is defined over an infinite time interval. However, in practice, we often need to focus on the response of system during a finite time interval. The Lyapunov asymptotic stability cannot ensure the desired performance, which introduces the study on finite-time stability. In [26], the problem of $H_{\infty}$ controller design for morphing aircraft with asynchronous switching is studied. The finite-time stability is analyzed by the aid of MDADT method and Lyapunov functional method. Simulation results demonstrate the effectiveness of the proposed method. To the best of the authors' knowledge, the problem of integrated fault detection and fault tolerant control for switched system with asynchronous switching has not been fully investigated yet, which motivated the research in the paper.

Inspired by the literatures above, the problem of integrated fault detection and fault tolerant control for switched system is studied in this paper to reduce the complexities and achieve optimal performance. It is obvious that the data missing will always exist in practical system; thus, the asynchronous switching caused by data missing is considered. Moreover, the finite-time stability is ensured to obtain better performance. The switched model of flight vehicle is given based on the nonlinear dynamic system of HiMAT at first. Then the fault detection filters and fault tolerant controllers are designed simultaneously and the parameters 
are given in the form of LMIs. The MDADT method and multiple Lyapunov functional method are combined to guarantee the finite time stability of closed-loop system. Simulation in the end demonstrates the effectiveness and superiority of proposed method.

The paper is organized as follows: The problem statement is shown in Section 2. In Section 3, the main results of the paper are given. The simulation is given to demonstrate the effectiveness of proposed method in Section 4, which is followed by the conclusion in Section 5 .

\section{Preliminaries and Problem Statement}

The flight vehicle considered in this paper is the HiMAT vehicle, which is an open-loop unstable unmanned flight vehicle applied in many fields. Based on [27], 20 uncoupled operating points of HiMAT are given, which can cover the nonlinear dynamics of whole flight envelope. The operating points will be depicted in Table 1 .

For simplicity and conciseness, the longitudinal model of the flight vehicle is taken into consideration to verify the problem of fault detection and fault tolerant control. Moreover, the stability and maneuverability depend on the short period motion. Therefore, we can obtain the longitudinal short period discrete-time switched model of HiMAT within full flight envelop by setting the sampling time of continuous-time model as $T$, which can be described as follows:

$$
\left\{\begin{array}{l}
x(k+1)=A_{i} x(k)+B_{i} u(k)+D_{i} d(k)+F_{i} f(k), \\
y(k)=C_{i} x(k),
\end{array}\right.
$$

where $x(k)=\left[\begin{array}{ll}x_{\alpha} & x_{q}\end{array}\right]^{T} \in R^{x}$ denotes the state vector, where $x_{\alpha}$ is the attack angle and $x_{q}$ is pitch rate; $y(k) \in R^{y}$ is the output vector; $u(k)=\left[\begin{array}{lll}\xi_{e} & \xi_{v} & \xi_{c}\end{array}\right]^{T} \in R^{u}$ denotes the input signal, where $\xi_{e}$, $\xi_{v}$, and $\xi_{c}$ denote the elevator, elevon, and canard deflection, respectively; $d(k) \in R^{d}$ is the external disturbance and $f(k) \in R^{f}$ denotes the fault signal; $d(k)$ and $f(k)$ belong to $L_{2}[0, \infty) . A_{i}, B_{i}, C_{i}, D_{i}$, and $F_{i}$ are system matrices with appropriate dimensions, where $i \in \Omega$ is the switching signal, and we have $\Omega=\{1,2, \ldots, n\}$, where $n>1$ is the number of subsystems.

In practical flight control systems, there always exists data missing because of network transformation. To simplify the proof, we suppose that the data missing exists in the channel from sensors to filters/controllers.

Thus, the measured output can be described as

$$
\tilde{y}(k)=\theta(k) y(k),
$$

where $\theta(k)$ is random variable to describe the data missing and takes value in finite set of $\{0,1\}$, which is a Bernoulli distributed white sequence and satisfies the following mathematical expectation values:

$$
\left\{\begin{array}{l}
\operatorname{Prob}\{\theta(k)=1\}=E\{\theta(k)\}=\rho, \\
\operatorname{Prob}\{\theta(k)=0\}=1-E\{\theta(k)\}=1-\rho, \\
\operatorname{Var}\{\theta(k)\}=E\left\{(\theta(k)-\rho)^{2}\right\}=\rho(1-\rho),
\end{array}\right.
$$

TABLE 1: The trim condition for operating points of HiMAT vehicle.

\begin{tabular}{lccc}
\hline Operating point & $\begin{array}{c}\text { Mach } \\
\text { number }\end{array}$ & Altitude $(\mathrm{m})$ & Angle of attack $\left(^{\circ}\right)$ \\
\hline 1 & 0.29 & 762.5 & 3.18 \\
2 & 0.40 & 762.5 & 1.49 \\
3 & 0.60 & 762.5 & 0.69 \\
4 & 0.50 & 1525 & 1.02 \\
5 & 0.40 & 3050 & 2.17 \\
6 & 0.70 & 3050 & 0.73 \\
7 & 0.40 & 6100 & 3.60 \\
8 & 0.60 & 6100 & 1.48 \\
9 & 0.70 & 6100 & 1.08 \\
10 & 1.40 & 6100 & 2.06 \\
11 & 0.70 & 7625 & 1.38 \\
12 & 0.90 & 7625 & 1.19 \\
13 & 1.20 & 7625 & 2.15 \\
14 & 0.90 & 9150 & 1.36 \\
15 & 0.80 & 10675 & 1.77 \\
16 & 0.70 & 12200 & 2.98 \\
17 & 0.90 & 12200 & 1.96 \\
18 & 1.20 & 12200 & 2.23 \\
19 & 1.40 & 12200 & 2.03 \\
20 & 0.68 & 13725 & 4.11 \\
\hline
\end{tabular}

where $\rho \in[0,1]$ is a constant value, which is called the data missing rate.

The fault detection system is composed of residual generator and residual evaluator. In this paper, we design the fault detection filters as follows to generate the residual signal.

$$
\left\{\begin{array}{l}
\hat{x}(k+1)=A_{i} \hat{x}(k)+B_{i} u(k)+L_{i}(\tilde{y}(k)-\hat{y}(k)), \\
\hat{y}(k)=\rho C_{i} \hat{x}(k), \\
r(k)=\tilde{y}(k)-\hat{y}(k),
\end{array}\right.
$$

where $\hat{x}(k) \in \mathbf{R}^{n_{x}}$ is the state of filters, $\hat{y}(k)$ denotes the estimated value of output signal, $r(k) \in \mathbf{R}^{n_{r}}$ is the residual signal, and $L_{i}$ are the parameters of the filters to be determined.

To ensure the stability and the transient performance under the condition with faults, the fault tolerant controllers are proposed. We define the tracking error of output signal as $\varepsilon(k)=g(k)-\tilde{y}(k)$; then the problem of controller design can be summarized as constructing flight control system and making sure that

$$
\lim _{k \longrightarrow \infty} \varepsilon(k)=0 .
$$

Define the integral of tracking error as

$$
v(k)=\sum_{i=0}^{k-1} \varepsilon(i)=\sum_{i=0}^{k-1}(g(i)-\tilde{y}(i)) .
$$

Thus, the fault tolerant controllers are proposed as follows to ensure that the system is robust to fault signal.

$$
u(k)=K_{1, i} \hat{x}(k)+K_{2, i} v(k),
$$

where $K_{1, i}$ and $K_{2, i}$ are the parameters of controllers to be designed. 
Because of the existence of network, the switching of filters/controllers will lag behind that of subsystems, which is called asynchronous switching. Thus, there will exist unmatched periods and matched periods in each subsystem. The energy of the switched system will increase during the unmatched periods and decrease during the matched periods, which will lead to performance degradation. The increase rate during the unmatched period of $i$ th subsystem is defined as $b_{i}$, the decay rate is set to be $a_{i}$, and the increase coefficient of energy function at the switching instant of $i$ th subsystem is $\mu_{i}$; the length of the unmatched period in $i$ th subsystem is $\Delta_{i}$.

Based on the statement above, define the error of measured vector as $e(k)=x(k)-\widehat{x}(k)$, the augment state vector as $\tilde{x}(k)=\left[\begin{array}{lll}x^{T}(k) & e^{T}(k) & v^{T}(k)\end{array}\right]^{T}$, the augment error as $\eta(k)=\left[r_{e}^{T}(k) \varepsilon^{T}(k)\right]^{T}$, and the augment disturbance as $\omega(k)=\left[\begin{array}{lll}d^{T}(k) & f^{T}(k) & h^{T}(k)\end{array}\right]^{T}$; then one can obtain the closed-loop system of flight vehicles as follows:

$$
\begin{aligned}
& \left\{\begin{array}{l}
\tilde{x}(k+1)=\widetilde{A}_{i i} \tilde{x}(k)+\widetilde{\theta}(k) \widetilde{A}_{1 i i} \tilde{x}(k)+\widetilde{B}_{i i} \omega(k), \\
\eta(k)=\widetilde{C}_{i i} \tilde{x}(k)+\widetilde{\theta}(k) \widetilde{C}_{1 i i} \tilde{x}(k)+\widetilde{D}_{i i} \omega(k), \\
\forall k \in\left[k_{i}+\Delta_{i}, k_{i+1}\right),
\end{array}\right. \\
& \left\{\begin{array}{l}
\tilde{x}(k+1)=\widetilde{A}_{i j} \tilde{x}(k)+\widetilde{\theta}(k) \widetilde{A}_{1 i j} \tilde{x}(k)+B_{i j} \omega(k), \\
\eta(k)=\widetilde{C}_{i j} \tilde{x}(k)+\widetilde{\theta}(k) \widetilde{C}_{1 i j} \tilde{x}(k)+\widetilde{D}_{i j} \omega(k), \\
\forall k \in\left[k_{i}, k_{i}+\Delta_{i}\right),
\end{array}\right.
\end{aligned}
$$

where $\tilde{\theta}(k)=\theta(k)-\rho ; \widetilde{A}_{i j}=\left[\begin{array}{ccc}A_{i}+B_{i} K_{1 j} & -B_{i} K_{1 j} & B_{i} K_{2 j} \\ \widetilde{A}_{i j}^{21} & \widetilde{A}_{i j}^{22} & \widetilde{A}_{i j}^{23} \\ -\rho C_{i} & 0 & I\end{array}\right]$; $\widetilde{A}_{1 i j}=\left[\begin{array}{ccc}0 & 0 & 0 \\ -L_{j} C_{i} & 0 & 0 \\ -C_{i} & 0 & 0\end{array}\right] ; \quad \widetilde{B}_{i j}=\left[\begin{array}{ccc}D_{i} & F_{i} & 0 \\ D_{i} & F_{i} & 0 \\ 0 & 0 & I\end{array}\right] ; \quad \widetilde{C}_{i j}=$ $\left[\begin{array}{ccc}0 & \rho C_{i} & 0 \\ -\rho C_{i} & 0 & 0\end{array}\right] ; \quad \widetilde{C}_{1 i j}=\left[\begin{array}{ccc}C_{i} & 0 & 0 \\ -C_{i} & 0 & 0\end{array}\right] ; \quad \widetilde{D}_{i j}=\left[\begin{array}{ccc}0 & -I & 0 \\ 0 & 0 & I\end{array}\right] ;$ $\left\{\begin{array}{l}\widetilde{A}_{i j}^{21}=A_{i}-A_{j}+\left[B_{i}-B_{j}\right] K_{1 j} \\ \widetilde{A}_{i j}^{22}=A_{j}-\left[B_{i}-B_{j}\right] K_{1 j}-\rho L_{j} C_{i} . \\ \widetilde{A}_{i j}^{23}=\left[B_{i}-B_{j}\right] K_{2 j}\end{array}\right.$

In this paper, the finite-time theory and switched control theory are combined to guarantee the stability and transient performance of the system. The following definitions and assumptions are introduced to simplify the proof.

Assumption 1. For given constant $N_{f}$, the external disturbance satisfies

$$
\sum_{k=0}^{N_{f}} \omega^{T}(k) \omega(k) \leq \bar{d}
$$

where $\bar{d} \geq 0$ is a given constant and denotes the upper bound of the disturbance in given time interval.

Definition 1 (see [26]) (finite-time stability, FTS). For given matrix $R>0$, we have positive constants $c_{1}, c_{2}$, and $N_{f}$ with $c_{1}<c_{2}$ and switching signal $\sigma(k)$. The closed-loop system (8) with $u(k) \equiv 0, d(k) \equiv 0$, and $f(k) \equiv 0$ is finite-time stable with respect to $\left(c_{1}, c_{2}, N_{f}, R, \sigma\right)$ if equation (10) holds.

$$
\tilde{x}^{T}\left(k_{0}\right) R \tilde{x}\left(k_{0}\right) \leq c_{1} \Longrightarrow \tilde{x}^{T}(k) R \tilde{x}(k) \leq c_{2}, \quad \forall k \in\left\{1, \ldots, N_{f}\right\} .
$$

Definition 2 (see [26]) (finite-time bounded, FTB). For given matrix $R>0$, we have positive constants $c_{1}, c_{2}$, and $N_{f}$ with $c_{1}<c_{2}$ and switching signal $\sigma(k)$. The closed-loop system (8) with external disturbance satisfying equation (9) is finite-time stable with respect to $\left(c_{1}, c_{2}, \bar{d}, N_{f}, R, \sigma\right)$ if equation (11) holds.

$$
\tilde{x}^{T}\left(k_{0}\right) R \tilde{x}\left(k_{0}\right) \leq c_{1} \Longrightarrow \tilde{x}^{T}(k) R \tilde{x}(k) \leq c_{2}, \quad \forall k \in\left\{1, \ldots, N_{f}\right\} .
$$

Definition 3 (see [26]) (finite-time $H_{\infty}$ performance). For given matrix $R>0$, we have positive constants $c_{2}, N_{f}$, and $\bar{d}$ and switching signal $\sigma(k)$. The closed-loop system $(8)$ is said to have finite-time $H_{\infty}$ performance if the system is finitetime bounded and equation (12) holds.

$$
\sum_{s=0}^{N_{f}} \eta^{T}(s) \eta(s) \leq \gamma_{w}^{2} \sum_{s=0}^{N_{f}} \omega^{T}(s) \omega(s)
$$

where $\gamma_{w}>0$ is prescribed performance.

The residual evaluation stage is composed of an evaluation function and a threshold. In this paper, the residual evaluation function and the threshold function are defined in equations (13) and (14).

$$
\begin{aligned}
J(k) & =\left(\frac{1}{L+1} \sum_{s=k-L}^{k} r^{T}(s) r(s)\right)^{1 / 2}, \\
J_{\text {th }} & =\sup _{\omega \in L_{2}(0, \infty), f=0} J(k),
\end{aligned}
$$

where $L$ is the length of evaluation time window. The decision can be made according to

$$
\begin{aligned}
& J(k)>J_{\text {th }} \Longrightarrow \text { faults } \Longrightarrow \text { alarm, } \\
& J(k)<J_{\text {th }} \Longrightarrow \text { no faults. }
\end{aligned}
$$

\section{Main Results}

In this section, the problems that integrated fault detection and fault tolerant will be considered and the parameters of the filters/controllers will be derived in the form of LMIs.

\subsection{Stability Analysis}

Definition 4 (see [26]). For a given switching signal $\sigma(k)$ and constants $k_{0}$ and $k_{1}$ with $k_{1}>k_{0} \geq 0$, define $N_{\sigma, i}\left(k_{0}, k_{1}\right)$ as the switching number of $i$ th subsystem during the time interval $\left(k_{0}, k_{1}\right)$. If equation (16) holds for given $N_{0, i} \geq 0$ and $\tau_{a i}>0$, then $N_{0 i}$ is called the mode-dependent chatter bounds and $\tau_{a i}$ is called the mode-dependent average dwell time, where 
$T_{i}\left(k_{0}, k_{1}\right)$ denotes the running time of $i$ th subsystem in the time interval $\left[k_{0}, k_{1}\right]$.

$$
N_{\sigma, i}\left(k_{0}, k_{1}\right) \leq N_{0, i}+\frac{T_{i}\left(k_{0}, k_{1}\right)}{\tau_{a i}} .
$$

Lemma 1 (see [26]). For given constants $0<a_{i}<1, b_{i}>0$, $c_{1}>0, c_{2}>0, \bar{d}>0, N_{f}>0, \mu_{1 i}>1, \mu_{2 i}>1$, and $\gamma>0$, if there exist matrices $S_{i}>0$ and $S_{i j}>0, \forall i, j \in \Omega, i \neq j$, such that

$$
\begin{aligned}
& S_{i} \leq \mu_{1 i} S_{i j}, \\
& S_{i j} \leq \mu_{2 i} S_{j} \text {, } \\
& {\left[\begin{array}{ccccc}
-S_{i} & 0 & \widetilde{A}_{i i} S_{i} & \widetilde{B}_{i i} \\
& * & -S_{i} & \bar{\rho} \widetilde{A}_{1 i i} S_{i} & 0 \\
* & * & -\left(1-a_{i}\right) S_{i} & 0 \\
* & * & * & -\gamma^{2} W_{i}
\end{array}\right]<0,} \\
& {\left[\begin{array}{cccc}
-S_{i j} & 0 & \widetilde{A}_{i j} S_{j} & \widetilde{B}_{i j} \\
* & -S_{i j} & \bar{\rho} \widetilde{A}_{1 i j} S_{j} & 0 \\
* & * & -\left(1+b_{i}\right) \widetilde{S}_{i j} & 0 \\
* & * & * & -\gamma^{2} W_{i}
\end{array}\right]}
\end{aligned}
$$

then the switched system with MDADT satisfying equations (18) and (19) is finite-time bounded with respect to $\left(c_{1}, c_{2}, \bar{d}, N_{f}, R, \sigma\right)$.

$$
\begin{gathered}
\tau_{a i} \geq \tau_{a i}^{*}=\frac{N_{f} \ln \mu_{1 i} \mu_{2 i}+N_{f} \Delta_{i} \ln \widetilde{\vartheta}_{i}}{\ln \left(c_{2} / \lambda_{1}\right)-\ln \left[\left(c_{1} / \lambda_{2}\right)+\gamma^{2} \widetilde{b}_{\max }^{N_{f}} \lambda_{3} \bar{d}\right]-N_{f} \ln \tilde{a}_{i}}, \\
\left(\frac{c_{1}}{\lambda_{2}}+\gamma^{2} \widetilde{b}_{\max }^{N_{f}} \lambda_{3} \bar{d}\right) \tilde{a}_{i}{ }^{N_{f}} \leq \frac{c_{2}}{\lambda_{1}}
\end{gathered}
$$

where $\bar{S}_{i j}=S_{i j}-S_{j}-S_{j}^{\mathrm{T}}, \quad \lambda_{1}=\max _{i \in \Omega}\left(\lambda_{\max }\left(\bar{S}_{i}\right), \lambda_{\max }\left(\bar{S}_{i j}\right)\right)$, $\lambda_{2}=\min _{i \in \Omega}\left(\lambda_{\min }\left(\bar{S}_{i}\right), \lambda_{\text {min }}\left(\bar{S}_{i j}\right)\right), \quad \lambda_{3}=\lambda_{\text {max }}\left(W_{i}\right), \quad \bar{S}_{i}=$ $R^{1 / 2} S_{i} R^{1 / 2}, \bar{S}_{i j}=R^{1 / 2} S_{i j} R^{1 / 2}, \tilde{a}_{i}=1-a_{i}, \widetilde{b}_{i}=1+b_{i}, \widetilde{\vartheta}_{i}=\left(\widetilde{b}_{i} / \widetilde{a}_{i}\right)$, and $\bar{\rho}=\sqrt{\rho(1-\rho)}$.

Lemma 2. For given matrix $C \in R^{n \times m}$ with rand $(C)=n$, we can obtain equation (20) by performing singular value decomposition to $C$ :

$$
C=U\left[\begin{array}{ll}
\Sigma & 0
\end{array}\right] E
$$

where $U \in \mathbf{R}^{n \times n}$ and $E \in \mathbf{R}^{m \times m}$ with $E=\left[\begin{array}{ll}E_{1}^{T} & E_{2}^{T}\end{array}\right]^{T}$, $E_{1} \in \mathbf{R}^{n \times m}$, and $E_{2} \in \mathbf{R}^{(m-n) \times m}$ are orthogonal matrices; $\Sigma=$ $\operatorname{diag}\left\{\lambda_{1}, \ldots, \lambda_{n}\right\}$ and $\lambda_{1}, \ldots, \lambda_{n}$ are nonzero singular values of $C$.
If there exists $\widehat{X} \in \mathrm{R}^{n \times n}$ satisfying equation (21), we can obtain $X \in \mathbf{R}^{m \times m}$, such that $\widehat{X} C=C X$, where $X=E_{1}^{T} X_{1} E_{1}$ and

$$
\widehat{X}=U \Sigma X_{1} \Sigma^{-1} U^{T}
$$

Proof. If $\widehat{X}=U \Sigma X_{1} \Sigma^{-1} U^{T}, C=U\left[\begin{array}{ll}\Sigma & 0\end{array}\right] E$, and $\widehat{X} C=C X$, we can obtain that

$$
\begin{aligned}
\widehat{X} C & =U \Sigma X_{1} \Sigma^{-1} U^{T} U\left[\begin{array}{ll}
\Sigma & 0
\end{array}\right] E \\
& =U \Sigma X_{1} \Sigma^{-1} U^{T} U\left[\begin{array}{ll}
\Sigma & 0
\end{array}\right]\left[\begin{array}{l}
E_{1} \\
E_{2}
\end{array}\right] \\
& =U \Sigma X_{1} E_{1}=C X=U\left[\begin{array}{ll}
\Sigma & 0
\end{array}\right]\left[\begin{array}{l}
E_{1} \\
E_{2}
\end{array}\right] X \\
& \Longleftrightarrow X=E_{1}^{T} X_{1} E_{1},
\end{aligned}
$$

which completes the proof.

Theorem 1. Given constants $0<a_{i}<1, b_{i}>0, c_{2}>0, \bar{d}>0$, $N_{f}>0, \mu_{1 i}>1, \mu_{2 i}>1$, and $\gamma>0$, if there exist matrices $S_{i}>0$ and $S_{i j}>0, \forall i, j \in \Omega, i \neq j$, such that

$$
\begin{gathered}
\text { S } \leq \mu_{1 i} S_{i j}, \\
{\left[\begin{array}{cccccc}
-S_{i} & 0 & 0 & 0 & \widetilde{A}_{i i} S_{i} & \widetilde{B}_{i i} \\
* & -S_{i} & 0 & 0 & \bar{\rho} \widetilde{A}_{1 i i} S_{i} & 0 \\
* & * & -I & 0 & \widetilde{C}_{i i} S_{i} & \widetilde{D}_{i i} \\
* & * & * & -I & \bar{\rho} \widetilde{C}_{1 i i} S_{i} & 0 \\
* & * & * & * & -\left(1-a_{i}\right) S_{i} & 0 \\
* & * & * & * & * & -\gamma^{2} I
\end{array}\right]<0,} \\
{\left[\begin{array}{cccccc}
-S_{i j} & 0 & 0 & 0 & \widetilde{A}_{i j} S_{j} & \widetilde{B}_{i j} \\
* & -S_{i j} & 0 & 0 & \bar{\rho} \widetilde{A}_{1 i j} S_{j} & 0 \\
* & * & -I & 0 & \widetilde{C}_{i j} S_{j} & \widetilde{D}_{i j} \\
* & * & * & -I & \bar{\rho}_{1 i j} S_{j} & 0 \\
* & * & * & * & -\left(1+b_{i}\right) \bar{S}_{i j} & 0 \\
* & * & * & * & * & -\gamma^{2} I
\end{array}\right]<0,}
\end{gathered}
$$

then the switched system (8) with MDADT satisfying equations (27) and (28) is finite-time stable with prescribed $H_{\infty}$ performance $\gamma_{w}$ with respect to $\left(0, c_{2}, \bar{d}, N_{f}, R, \sigma, \gamma_{w}\right)$.

$$
\begin{gathered}
\tau_{a i} \geq \tau_{a i}^{*}=\max \left\{\frac{N_{f} \ln \mu_{1 i} \mu_{2 i}+N_{f} \Delta_{i} \ln \widetilde{\vartheta}_{i}}{\ln \left(c_{2} / \lambda_{1}\right)-\ln \left[\gamma^{2} \widetilde{b}_{\max }^{N_{f}} \bar{d}\right]-N_{f} \ln \tilde{a}_{i}}, \frac{\Delta_{i} \ln \widetilde{\vartheta}_{i}+\ln \mu_{1 i} \mu_{2 i}}{-\ln \tilde{a}_{i}}\right\}, \\
\left(\gamma^{2} \widetilde{b}_{\max }^{N_{f}} \bar{d}\right) \tilde{a}_{i}^{N_{f}} \leq \frac{c_{2}}{\lambda_{1}}
\end{gathered}
$$


where $\quad \lambda_{1}=\max _{i \in \Omega}\left(\lambda_{\max }\left(\bar{S}_{i}\right), \lambda_{\max }\left(\bar{S}_{i j}\right)\right), \quad \lambda_{2}=$ $\min _{i \in \Omega}\left(\lambda_{\min }\left(\bar{S}_{i}\right), \lambda_{\min }\left(\bar{S}_{i j}\right)\right), \bar{\rho}=\sqrt{\rho(1-\rho)}, \bar{S}_{i}=R^{1 / 2} S_{i} R^{1 / 2}$, $\tilde{a}_{i}=1-a_{i}, \quad \widetilde{b}_{i}=1+b_{i}, \quad \widetilde{\vartheta}_{i}=\left(\widetilde{b}_{i} / \widetilde{a}_{i}\right), \quad$ and $\quad \gamma_{w}=$ $\gamma\left(\tilde{a}_{\max } / \tilde{a}_{\min }\right)^{N_{f} / 2}$.

Proof. The Lyapunov functions of the switched system are defined as follows:

$$
\left\{\begin{array}{l}
V_{i}(k)=\tilde{x}^{T}(k) P_{i} \widetilde{x}(k), \quad \forall k \in\left[k_{i}+\Delta_{i}, k_{i+1}\right), \\
V_{i j}(k)=\tilde{x}^{T}(k) P_{i j} \tilde{x}(k), \quad \forall k \in\left[k_{i}, k_{i}+\Delta_{i}\right),
\end{array}\right.
$$

where $P_{i}>0, P_{i j}>0$.

Let $\varsigma(k)=\left[\begin{array}{lll}\widetilde{x}^{T}(k) & \omega^{T}(k)\end{array}\right]^{T}$. Under zero initial conditions, we can obtain that

$$
\begin{aligned}
& \Delta V_{i}(k)+a_{i} V_{i}(k)+\mathrm{E}\left\{\eta^{T}(k) \eta(k)\right\}-\gamma^{2} \omega^{T}(k) \omega(k) \\
& =\mathrm{E}\left\{\tilde{x}(k+1)^{T} P_{i} \widetilde{x}(k+1)\right\}-\widetilde{x}^{T}(k)\left(1-a_{i}\right) P_{i} \tilde{x}(k)+\mathrm{E}\left\{\eta^{T}(k) \eta(k)\right\}-\gamma^{2} \omega^{T}(k) \omega(k) \\
& =\varsigma^{T}(k)\left[\begin{array}{c}
\widetilde{A}_{i i}^{T}+\widetilde{\theta}(k) \widetilde{A}_{1 i i}^{T} \\
\widetilde{B}_{i i}^{T}
\end{array}\right] P_{i}\left[\widetilde{A}_{i i}+\widetilde{\theta}(k) \widetilde{A}_{1 i i} \widetilde{B}_{i i}\right] \varsigma(k) \\
& +\varsigma^{T}(k)\left[\begin{array}{c}
\widetilde{C}_{i i}^{T}+\widetilde{\theta}(k) \widetilde{C}_{1 i i}^{T} \\
\widetilde{D}_{i i}^{T}
\end{array}\right]\left[\widetilde{C}_{i i}\left(\rho_{k}\right)+\tilde{\theta}(k) \widetilde{C}_{1 i i} \widetilde{D}_{i i}\right] \varsigma(k) \\
& =\varsigma^{T}(k)\left\{\left[\begin{array}{c}
\widetilde{A}_{i i}^{T} \\
\widetilde{B}_{i i}^{T}
\end{array}\right] P_{i}\left[\begin{array}{ll}
\widetilde{A}_{i i} & \widetilde{B}_{i i}
\end{array}\right]+\bar{\rho}^{2}\left[\begin{array}{c}
\widetilde{A}_{1 i i}^{T} \\
0
\end{array}\right] P_{i}\left[\begin{array}{ll}
\widetilde{A}_{1 i i} & 0
\end{array}\right]+\left[\begin{array}{c}
\widetilde{C}_{i i}^{T} \\
\widetilde{D}_{i i}^{T}
\end{array}\right]\left[\begin{array}{ll}
\widetilde{C}_{i i}, & \widetilde{D}_{i i}
\end{array}\right]\right. \\
& \left.+\bar{\rho}^{2}\left[\begin{array}{c}
\widetilde{C}_{1 i i}^{T} \\
0
\end{array}\right]\left[\widetilde{C}_{1 i i}, 0\right]+\left[\begin{array}{cc}
-\left(1-a_{i}\right) P_{i} & 0 \\
0 & -\gamma^{2} I
\end{array}\right]\right\} \varsigma(k) \\
& =\varsigma^{T}(k) Z_{i i} \varsigma(k) \text {. }
\end{aligned}
$$

Similarly, we can conclude that

$$
\begin{aligned}
& \Delta V_{i}(k)-b_{i} V_{i}(k)+\mathrm{E}\left\{\eta^{T}(k) \eta(k)\right\}-\gamma^{2} \omega^{T}(k) \omega(k) \\
& =\mathrm{E}\left\{\tilde{x}(k+1)^{T} P_{i j} \tilde{x}(k+1)\right\}-\widetilde{x}^{T}(k)\left(1+b_{i}\right) P_{i j} \tilde{x}(k)+\mathrm{E}\left\{\eta^{T}(k) \eta(k)\right\}-\gamma^{2} \omega^{T}(k) \omega(k) \\
& =\varsigma^{T}(k)\left[\begin{array}{c}
\tilde{A}_{i j}^{T}+\tilde{\theta}(k) \tilde{A}_{1 i j}^{T} \\
\widetilde{B}_{i j}^{T}
\end{array}\right] P_{i j}\left[\widetilde{A}_{i j}+\tilde{\theta}(k) \widetilde{A}_{1 i j} \widetilde{B}_{i j}\right] \varsigma(k) \\
& +\varsigma^{T}(k)\left[\begin{array}{c}
\widetilde{C}_{i j}^{T}+\widetilde{\theta}(k) \widetilde{C}_{1 i j}^{T} \\
\widetilde{D}_{i j}^{T}
\end{array}\right]\left[\widetilde{C}_{i j}+\widetilde{\theta}(k) \widetilde{C}_{1 i j} \widetilde{D}_{i j}\right] \varsigma(k) \\
& =\varsigma^{T}(k)\left\{\left[\begin{array}{c}
\widetilde{A}_{i j}^{T} \\
\widetilde{B}_{i j}^{T}
\end{array}\right] P_{i j}\left[\widetilde{A}_{i j}, \widetilde{B}_{i j}\right]+\bar{\rho}^{2}\left[\begin{array}{c}
\widetilde{A}_{1 i j}^{T} \\
0
\end{array}\right] P_{i j}\left[\widetilde{A}_{1 i j}, 0\right]+\left[\begin{array}{c}
\widetilde{C}_{i j}^{T} \\
\widetilde{D}_{i j}^{T}
\end{array}\right]\left[\begin{array}{ll}
\widetilde{C}_{i j}, & \widetilde{D}_{i j}
\end{array}\right]\right. \\
& \left.+\bar{\rho}^{2}\left[\begin{array}{c}
\widetilde{C}_{1 i j}^{T} \\
0
\end{array}\right]\left[\widetilde{C}_{1 i j}, 0\right]+\left[\begin{array}{cc}
-\left(1+b_{i}\right) P_{i j} & 0 \\
0 & -\gamma^{2} I
\end{array}\right]\right\} \varsigma(k) \\
& =\varsigma^{T}(k) Z_{i j} \varsigma(k) \text {. }
\end{aligned}
$$

Define $S_{i}=P_{i}^{-1}$ and make a congruence transformation to equation (25) by $\operatorname{diag}\left\{S_{i}^{-1}, S_{i}^{-1}, I, I, S_{i}^{-1}, I\right\}$; then we can obtain equation (32). Similarly, one can obtain equation (33) by making a congruence transformation to equation (26). 


$$
\begin{gathered}
{\left[\begin{array}{cccccc}
-P_{i} & 0 & 0 & 0 & P_{i} \widetilde{A}_{i i} & P_{i} \widetilde{B}_{i i} \\
* & -P_{i} & 0 & 0 & \bar{\rho} P_{i} \widetilde{A}_{1 i i} & 0 \\
* & * & -I & 0 & \widetilde{C}_{i i} & \widetilde{D}_{i i} \\
* & * & * & -I & \bar{\rho} \widetilde{C}_{1 i i} & 0 \\
* & * & * & * & -\left(1-a_{i}\right) P_{i} & 0 \\
* & * & * & * & * & -\gamma^{2} I
\end{array}\right]<0,} \\
{\left[\begin{array}{cccccc}
-P_{i j} & 0 & 0 & 0 & P_{i j} \widetilde{A}_{i j} & P_{i j} \widetilde{B}_{i j} \\
* & -P_{i j} & 0 & 0 & \bar{\rho} P_{i j} \widetilde{A}_{1 i j} & 0 \\
* & * & -I & 0 & \widetilde{C}_{i j} & \widetilde{D}_{i j} \\
* & * & * & -I & \bar{\rho} \widetilde{C}_{1 i j} & 0 \\
* & * & * & * & -\left(1+b_{i}\right) P_{i j} & 0 \\
* & * & * & * & * & -\gamma^{2} I
\end{array}\right]<0 .}
\end{gathered}
$$

By the aid of Schur component, we can conclude that equation (32) is equivalent to $Z_{i i}<0$, and equation (33) is equivalent to $Z_{i j}<0$; that is,

$$
\Delta V_{i}(k) \leq \begin{cases}-a_{i} V_{i}(k)-E\left\{\eta^{T}(k) \eta(k)\right\}+\gamma^{2} \omega^{T}(k) \omega(k), & \forall k \in\left[k_{i}+\Delta_{i}, k_{i+1}\right), \\ b_{i} V_{i}(k)-E\left\{\eta^{T}(k) \eta(k)\right\}+\gamma^{2} \omega^{T}(k) \omega(k), & \forall k \in\left[k_{i}, k_{i}+\Delta_{i}\right) .\end{cases}
$$

Similar to the proof in Theorem 1 , we can obtain equation (35) by setting $\gamma^{2} \omega^{T}(k) W_{i} \omega(k)$ as $\psi(s)=-\mathrm{E}\left\{\eta^{T}(k) \eta(k)\right\}+\gamma^{2} \omega^{T}(k) \omega(k)$. Moreover, the switched system (8) is finite-time bounded with respect to $\left(0, c_{2}, \bar{d}, N_{f}, R, \sigma\right)$ by setting $W_{i}=I$ and $c_{1}=0$ if equations (23)-(28) are satisfied.

$$
\begin{aligned}
V_{\sigma(k)}(k) \leq & \prod_{i=1}^{n} \mu_{1 i}^{N_{\sigma, i}\left(k_{0}, k\right)} \mu_{2 i}^{N_{\sigma, i}\left(k_{0}, k\right)} \widetilde{a}_{i}^{T_{i}\left(k_{0}, k\right)} \widetilde{\vartheta}_{i}^{\Delta_{i} N_{\sigma, i}\left(k_{0}, k\right)} V_{\sigma\left(k_{0}\right)}\left(k_{0}\right) \\
& +\widetilde{b}_{\max }^{N_{f}} \sum_{s=k_{0}}^{k-1}\left(\prod_{i=1}^{n} \mu_{1 i}^{N_{\sigma, i}(s, k)} \mu_{2 i}^{N_{\sigma, i}(s, k)} \tilde{a}_{i}^{T_{i}(s, k)} \widetilde{\vartheta}_{i}^{\Delta_{i} N_{\sigma, i}(s, k)}\right) \psi(s) .
\end{aligned}
$$

Together with the fact that $V_{\sigma(k)}(k) \geq 0$, we can conclude that

$$
\begin{aligned}
& \sum_{s=k_{0}}^{k-1}\left(\prod_{i=1}^{n} \mu_{1 i}^{N_{\sigma, i}(s, k)} \mu_{2 i}^{N_{\sigma, i}(s, k)} \tilde{a}_{i}^{T_{i}(s, k)} \widetilde{\vartheta}_{i}^{\Delta_{i} N_{\sigma, i}}\right) \psi(s, k) \geq 0 \\
& \Longleftrightarrow \sum_{s=k_{0}}^{k-1}\left(\prod_{i=1}^{n} \mu_{1 i}^{N_{\sigma, i}(s, k)} \mu_{2 i}^{N_{\sigma, i}(s, k)} \tilde{a}_{i}^{T_{i}(s, k)} \widetilde{\vartheta}_{i}^{\Delta_{i} N_{\sigma, i}(s, k)}\right) \eta^{T}(k) \eta(k) \\
& \leq \gamma^{2} \sum_{s=k_{0}}^{k-1}\left(\prod_{i=1}^{n} \mu_{1 i}^{N_{\sigma, i}(s, k)} \mu_{2 i}^{N_{\sigma, i}(s, k)} \tilde{a}_{i}^{T_{i}(s, k)} \widetilde{\vartheta}_{i}^{\Delta_{i} N_{\sigma, i}(s, k)}\right) \omega^{T}(s) \omega(s) .
\end{aligned}
$$

Multiplying both sides of equation (36) by $\prod_{i=1}^{n}\left(\widetilde{\vartheta}_{i}^{\Delta_{i}} \mu_{1 i} \mu_{2 i}\right)^{-N_{\sigma i}\left(k_{0}, k\right)}$, one can obtain that

$$
\begin{aligned}
& \sum_{s=k_{0}}^{k-1}\left(\prod_{i=1}^{n} \mu_{1 i}^{-N_{\sigma, i}\left(k_{0}, s\right)} \mu_{2 i}^{-N_{\sigma, i}\left(k_{0}, s\right)} \tilde{a}_{i}^{T_{i}(s, k)} \widetilde{\vartheta}_{i}^{-\Delta_{i} N_{\sigma, i}\left(k_{0}, s\right)}\right) \eta^{T}(k) \eta(k) \\
& \leq \gamma^{2} \sum_{s=k_{0}}^{k-1}\left(\prod_{i=1}^{n} \mu_{1 i}^{-N_{\sigma, i}\left(k_{0}, s\right)} \mu_{2 i}^{-N_{\sigma, i}\left(k_{0}, s\right)} \tilde{a}_{i}^{T_{i}(s, k)} \widetilde{\vartheta}_{i}^{-\Delta_{i} N_{\sigma, i}\left(k_{0}, s\right)}\right) \\
& \cdot \omega^{T}(s) \omega(s) .
\end{aligned}
$$

Together with equations (27) and (28), we have

$$
0 \leq N_{\sigma, i}\left(k_{0}, s\right) \leq \frac{T_{i}\left(k_{0}, s\right)}{\tau_{a i}^{*}} \leq \frac{-T_{i}\left(k_{0}, s\right) \ln \tilde{a}_{i}}{\Delta_{i}\left(\ln \widetilde{b}_{i}-\ln \widetilde{a}_{i}\right)+\ln \mu_{1 i} \mu_{2 i}} .
$$

According to equations (37) and (38), we have

$$
\begin{aligned}
& \sum_{s=k_{0}}^{k-1}\left(\prod_{i=1}^{n}\left(\mu_{1 i} \mu_{2 i} \widetilde{\vartheta}_{i}^{\Delta_{i}}\right)^{\left(T_{i}\left(k_{0}, s\right) \ln \tilde{a}_{i}\left(\Delta_{i}\left(\ln \tilde{b}_{i}-\ln \tilde{a}_{i}\right)+\ln \mu_{1 i} \mu_{2 i}\right)\right)} \tilde{a}_{i}^{T_{i}(s, k)}\right) \\
& \cdot \eta^{T}(k) \eta(k) \\
& \leq \gamma^{2} \sum_{s=k_{0}}^{k-1}\left(\prod_{i=1}^{n}\left(\mu_{1 i} \mu_{2 i} \widetilde{\vartheta}_{i}^{\Delta_{i}}\right)^{\left(T_{i}\left(k_{0}, s\right) \ln \tilde{a}_{i} /\left(\Delta_{i}\left(\ln \tilde{b}_{i}-\ln \tilde{a}_{i}\right)+\ln \mu_{1 i} \mu_{2 i}\right)\right)} \tilde{a}_{i}^{T_{i}(s, k)}\right) \\
& \cdot \omega^{T}(s) \omega(s) .
\end{aligned}
$$

Moreover, it is noted that $\left(\mu_{1 i} \mu_{2 i} \widetilde{\vartheta}_{i}^{\Delta_{i}}\right)^{\left(T_{i}\left(k_{0}, s\right) \ln \tilde{a}_{i} /\left(\Delta_{i}\left(\ln \tilde{b}_{i}-\ln \tilde{a}_{i}\right)+\ln \mu_{1 i} \mu_{2 i}\right)\right)}=\tilde{a}_{i}^{T_{i}\left(k_{0}, s\right)}$. Thus, we can conclude that 


$$
\begin{aligned}
& \sum_{s=k_{0}}^{k-1}\left(\prod_{i=1}^{n} \tilde{a}_{i}^{T_{i}\left(k_{0}, k\right)}\right) \eta^{T}(k) \eta(k) \\
& \quad \leq \gamma^{2} \sum_{s=k_{0}}^{k-1}\left(\prod_{i=1}^{n} \tilde{a}_{i}^{T_{i}\left(k_{0}, k\right)}\right) \omega^{T}(s) \omega(s) .
\end{aligned}
$$

Let $k-1=N_{f}$; we have

$$
\begin{aligned}
& \sum_{s=k_{0}}^{N_{f}}\left(\prod_{i=1}^{n} \tilde{a}_{i}^{T_{i}\left(k_{0}, k\right)}\right) \eta^{T}(k) \eta(k) \\
& \leq \gamma^{2} \sum_{s=k_{0}}^{k-1}\left(\prod_{i=1}^{n} \tilde{a}_{i}^{T_{i}\left(k_{0}, k\right)}\right) \omega^{T}(s) \omega(s) \\
& \Longleftrightarrow \widetilde{a}_{\min }^{N_{f}} \sum_{s=k_{0}}^{N_{f}} \eta^{T}(k) \eta(k) \leq \gamma^{2} \widetilde{a}_{\max }^{N_{f}} \sum_{s=k_{0}}^{k-1} \omega^{T}(s) \omega(s) \\
& \Longleftrightarrow \sum_{s=k_{0}}^{N_{f}} \eta^{T}(k) \eta(k) \leq \gamma^{2}\left(\frac{\widetilde{a}_{\max }}{\widetilde{a}_{\min }}\right)^{N_{f}} \sum_{s=k_{0}}^{k-1} \omega^{T}(s) \omega(s) .
\end{aligned}
$$

Based on the above statement, we can obtain the closedloop switched system (8) which is finite-time bounded with prescribed $H_{\infty}$ performance $\gamma_{w}=\gamma\left(\tilde{a}_{\max } / \tilde{a}_{\text {min }}\right)^{N_{f} / 2}$.

The sufficient conditions to guarantee that the system is finite-time bounded with prescribed performance are given in Theorem 1, and the solutions of the controllers and filters will be given in Theorem 2 .

\subsection{Controllers and Filters Design}

Theorem 2. Consider the switched system (8) and constant parameters $0<a_{i}<1, b_{i}>0, c_{2}>0, \bar{d}>0, N_{f}>0, \mu_{1 i}>1$, $\mu_{2 i}>1$, and $\gamma>0$. If there exist matrices $S_{i}>0$ and $S_{i j}>0$, $\forall i, j \in \Omega, i \neq j$, such that

$$
\begin{gathered}
\text { S } \leq \mu_{1 i} S_{i j}, \\
{\left[\begin{array}{cccccc}
\varphi_{i i}^{11} & 0 & 0 & 0 & \varphi_{i i}^{15} & \varphi_{i i}^{16} \\
* & \varphi_{i i}^{22} & 0 & 0 & \varphi_{i i}^{25} & 0 \\
* & * & -I & 0 & \widetilde{C}_{i i} \varphi_{i i}^{35} & 0 \\
* & * & * & -I & \widetilde{C}_{1 i i} \varphi_{i i}^{45} & \widetilde{D}_{i i} \\
* & * & * & * & \varphi_{i i}^{55} & 0 \\
* & * & * & * & * & -\gamma^{2} I
\end{array}\right]<0,} \\
{\left[\begin{array}{cccccc}
\varphi_{i j}^{11} & 0 & 0 & 0 & \varphi_{i j}^{15} & \varphi_{i j}^{16} \\
* & \varphi_{i j}^{22} & 0 & 0 & \varphi_{i j}^{25} & 0 \\
* & * & -I & 0 & \widetilde{C}_{i j} \varphi_{i j}^{35} & \widetilde{D}_{i j} \\
* & * & * & -I & \widetilde{\rho}_{1 i j} \varphi_{i j}^{45} & 0 \\
* & * & * & * & \varphi_{i j}^{55} & 0 \\
* & * & * & * & * & -\gamma^{2} I
\end{array}\right]<0,}
\end{gathered}
$$

then the switched system (8) with MDADT satisfying (27) and (28) is finite-time bounded with prescribed $H_{\infty}$ performance, and the parameters of the controllers and filters can be given as

$$
\begin{gathered}
K_{1, i}=\bar{K}_{1, i} S_{1, i}^{-1}=\bar{K}_{1, i}\left(E_{i}^{T} X_{11 i} E_{i}\right)^{-1}, \\
K_{2, i}=\bar{K}_{2, i} S_{3, i}^{-1}, \\
L_{i}=\bar{L}_{i} \widehat{S}_{1, i}^{-1}=\bar{L}_{i}\left(U_{i} \Sigma_{i} X_{1, i} \Sigma_{i}^{-1} U_{i}^{T}\right)^{-1},
\end{gathered}
$$

where $\varphi_{i i}^{11}=\varphi_{i i}^{22}=-S_{i}, \varphi_{i j}^{11}=\varphi_{i j}^{22}=-S_{i j}, \varphi_{i i}^{66}=-\left(1-a_{i}\right) S_{i}$, $\varphi_{i j}^{66}=-\left(1+b_{i}\right)\left[S_{i j}-S_{j}-S_{j}^{T}\right]$,

$\varphi_{i j}^{15}=\left[\begin{array}{ccc}A_{i} S_{1, j}+B_{i} \bar{K}_{1, j} & -B_{i} \bar{K}_{1, j} & B_{i} \bar{K}_{2, j} \\ \bar{\varphi}_{i j}^{21} & \bar{\varphi}_{i j}^{22} & \bar{\varphi}_{i j}^{23} \\ -\rho C_{i} S_{1, j} & 0 & S_{3, j}^{T}\end{array}\right]$,

$\bar{\varphi}_{i j}^{21}=\left[A_{i}-A_{j}\right] S_{1, j}+\left[B_{i}-B_{j}\right] \bar{K}_{1, j}, \quad \bar{\varphi}_{i j}^{22}=A_{j} S_{2, j}-\left[B_{i}-B_{j}\right]$

$\bar{K}_{1, j}-\rho \bar{L}_{j} C_{i}, \quad \bar{\varphi}_{i j}^{23}=\left[B_{i}-B_{j}\right] \bar{K}_{2, j}, \quad \varphi_{i j}^{25}=\bar{\rho}\left[\begin{array}{ccc}0 & 0 & 0 \\ -\bar{L}_{j} C_{i} & 0 & 0 \\ -C_{i} S_{1, j} & 0 & 0\end{array}\right]$,

$\varphi_{i j}^{16}=\left[\begin{array}{ccc}D_{i} & F_{i} & 0 \\ D_{i} & F_{i} & 0 \\ 0 & 0 & I\end{array}\right], \quad \varphi_{i j}^{35}=\left[\begin{array}{ccc}0 & \rho C_{i} S_{2, j} & 0 \\ -\rho C_{i} S_{1, j} & 0 & 0\end{array}\right], \quad$ and $\varphi_{i j}^{45}=\left[\begin{array}{ccc}C_{i} S_{1, j} & 0 & 0 \\ -C_{i} S_{1, j} & 0 & 0\end{array}\right] . E_{i}$ and $U_{i}=\left[\begin{array}{ll}U_{1, i} & U_{2, i}\end{array}\right]$ can be given by the singular value decomposition for $C_{i}$. We have $C_{i}=$ $U_{i}\left[\begin{array}{ll}\Sigma_{i} & 0\end{array}\right] E_{i}$ and $S_{i}=\operatorname{diag}\left\{S_{1, i}, S_{2, i}, S_{3, i}\right\}$.

Proof. It is supposed that equation (49) can be obtained by singular value decomposition:

$$
C_{i}=U_{i}\left[\begin{array}{ll}
\Sigma_{i} & 0
\end{array}\right] E_{i},
$$

where $U_{i}$ and $E_{i}$ are orthogonal matrices.

Thus, based on Lemma 2, one can obtain that there exist matrices $S_{1, i}^{T}$ and $\widehat{S}_{1, i}^{T}$, such that

$$
\widehat{S}_{1, i}^{T} C_{i}=C_{i} S_{1, i}^{T} \text {, }
$$

where $S_{1, i}^{T}=E_{1 i}^{T} X_{1 i} E_{1 i}$ and $\widehat{S}_{1, i}^{T}=U_{i} \Sigma_{i} X_{1 i} \Sigma_{i}^{-1} U_{i}^{T}$.

Substitute (8) into (25) and let $\bar{K}_{1, i}=K_{1, i} S_{1, i}$, $\bar{K}_{2, i}=K_{2, i} S_{3, i}$, and $\bar{L}_{i}=L_{i} \widehat{S}_{1, i}$; thus we have (44). Similarly, substituting the system matrices into (26), we have (45). The parameters of the controllers and filters can be achieved by $\bar{K}_{1, i}, \bar{K}_{2, i}$, and $\bar{L}_{i}$ through (46)-(48), which completes the proof.

Remark 1. Compared to [26], the integrated fault detection and control are investigated. Because the fault detection filter and controller will influence each other, the method proposed in this article will overcome the undesirable response caused by the separated design of fault detection filter and controller, which can ensure optimal performance and avoid designing trial and error.

\section{Numerical Example}

The longitudinal motion dynamics of the vehicle can be modeled as a switched system. Define the sampling time as 
$T=0.02 \mathrm{~s}$, and the flight trajectory in the subsystems is set to be 19-18-12-9-8-4-2-1. Based on the nonlinear model of flight vehicle and data in [25], we can obtain the system matrices and input matrices of each subsystem as follows:

$$
\begin{aligned}
& A_{1}=\left[\begin{array}{ll}
0.9804 & 0.0188 \\
0.1768 & 0.9720
\end{array}\right], \\
& B_{1}=\left[\begin{array}{lll}
-0.0049 & -0.0034 & 0.0007 \\
-0.1579 & -0.0979 & 0.0993
\end{array}\right] \text {, } \\
& A_{2}=\left[\begin{array}{ll}
0.9728 & 0.0188 \\
0.3773 & 0.9622
\end{array}\right] \text {, } \\
& B_{2}=\left[\begin{array}{lll}
-0.0075 & -0.0050 & 0.0014 \\
-0.2941 & -0.1765 & 0.1831
\end{array}\right] \text {, } \\
& A_{4}=\left[\begin{array}{ll}
0.9688 & 0.0187 \\
0.4968 & 0.9560
\end{array}\right] \text {, } \\
& B_{4}=\left[\begin{array}{lll}
-0.0096 & -0.0065 & 0.0021 \\
-0.4334 & -0.2895 & 0.2547
\end{array}\right] \text {, } \\
& A_{8}=\left[\begin{array}{ll}
0.9766 & 0.0190 \\
0.3312 & 0.9668
\end{array}\right] \text {, } \\
& B_{8}=\left[\begin{array}{rrr}
-0.0077 & -0.0054 & 0.0018 \\
-0.3759 & -0.2798 & 0.2113
\end{array}\right] \text {, } \\
& A_{9}=\left[\begin{array}{ll}
0.9725 & 0.0189 \\
0.3344 & 0.9594
\end{array}\right], \\
& B_{9}=\left[\begin{array}{lll}
-0.0099 & -0.0068 & 0.0026 \\
-0.5374 & -0.3793 & 0.2890
\end{array}\right] \text {, } \\
& A_{12}=\left[\begin{array}{ll}
0.9649 & 0.0188 \\
0.2242 & 0.9509
\end{array}\right] \text {, } \\
& B_{12}=\left[\begin{array}{lll}
-0.0136 & -0.0094 & 0.0042 \\
-0.9015 & -0.6166 & 0.4367
\end{array}\right] \text {, } \\
& A_{18}=\left[\begin{array}{cc}
0.9657 & 0.0191 \\
-0.9772 & 0.9523
\end{array}\right] \text {, } \\
& B_{18}=\left[\begin{array}{lll}
-0.0061 & -0.0033 & 0.0023 \\
-0.4595 & -0.2426 & 0.2576
\end{array}\right] \text {, } \\
& A_{19}=\left[\begin{array}{cc}
0.9635 & 0.0192 \\
-1.2369 & 0.9507
\end{array}\right] \text {, } \\
& B_{19}=\left[\begin{array}{lll}
-0.0066 & -0.0032 & 0.0019 \\
-0.5334 & -0.2569 & 0.2163
\end{array}\right] \text {. }
\end{aligned}
$$

In order to illustrate the effectiveness of the proposed method, the fault signal $f$ is set to be a constant bias fault of elevator $\xi_{e}$. Thus, the fault signal can be described as
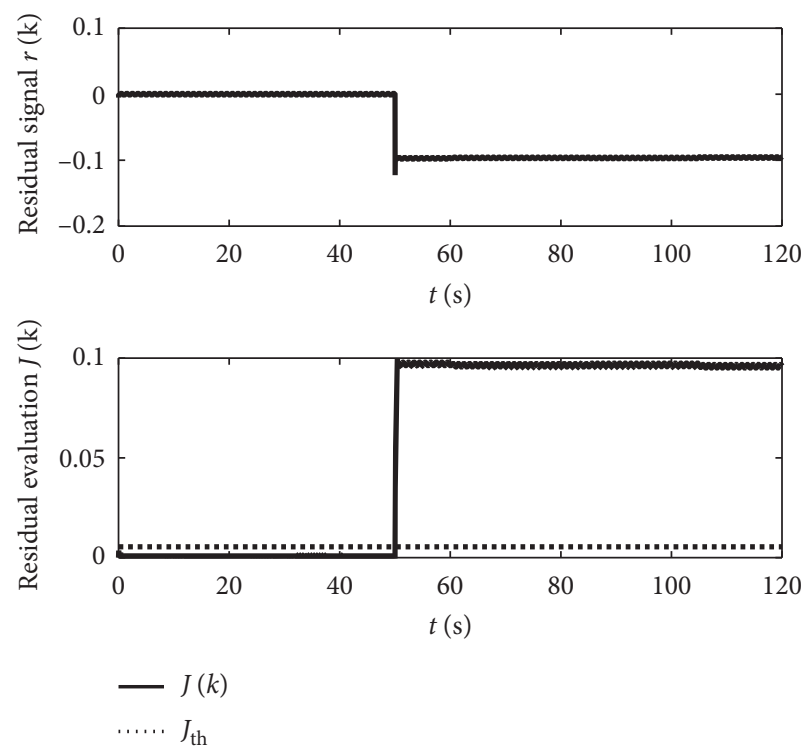

Figure 1: Residual signal and residual evaluation.

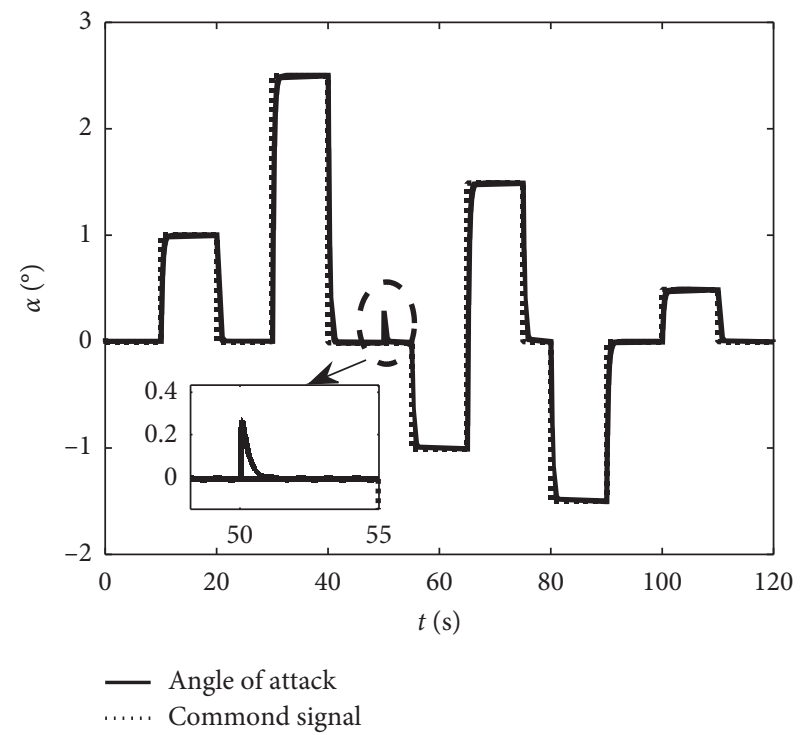

Figure 2: Response of attack angle.

$$
f= \begin{cases}0, & t<50 \mathrm{~s} \\ -0.1 \mathrm{rad}, & t \geq 50 \mathrm{~s} .\end{cases}
$$

In addition, the external disturbance is set to be harmonics wind gust, which can be generated as follows:

$$
\left\{\begin{array}{l}
l(k+1)=\left[\begin{array}{cc}
0.9922 & 0.1247 \\
-0.1247 & 0.9922
\end{array}\right] l(k), \\
d(k)=\left[\begin{array}{ll}
1 & 0
\end{array}\right] l(k),
\end{array}\right.
$$

where $l(k)$ denotes the state vector, whose initial value is set to be $[0.01 ; 0]$.

The probability of packet dropouts from output to filter/ controller is set to be $\rho=0.95$, the maximum allowable 


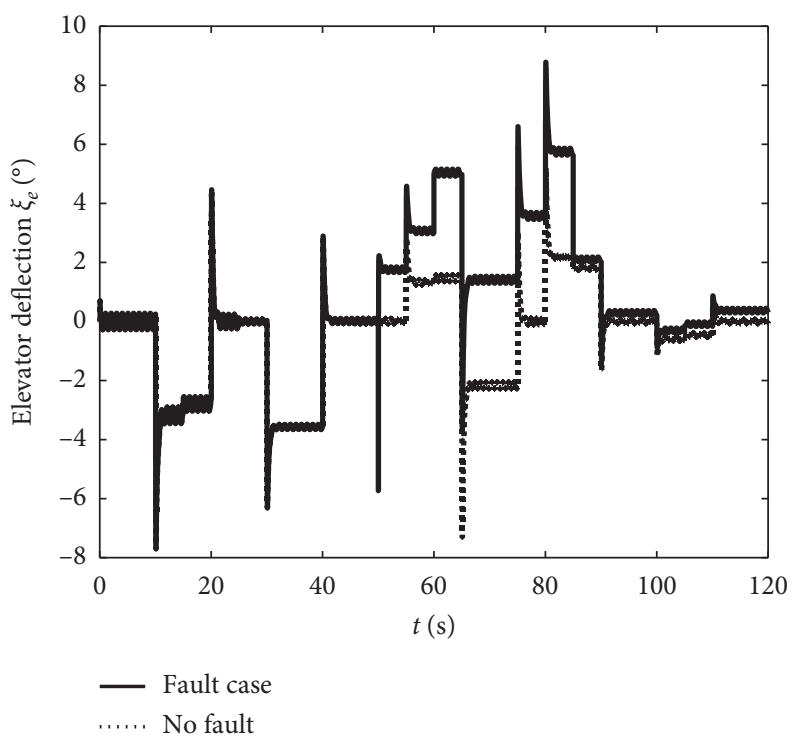

Figure 3: Response of elevator deflection.

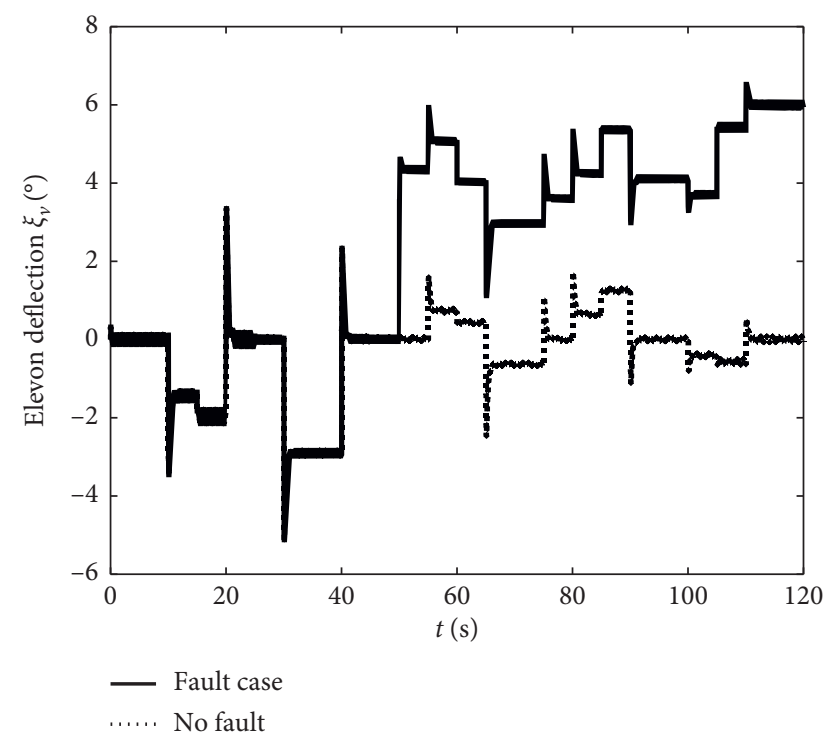

Figure 4: Response of elevon deflection.

upper bound of packet dropouts is set to be $\bar{N}_{1}=5$, the length of time window $N_{f}=25$, upper bound of the disturbance $\bar{d}=5$, and performance attenuation $\gamma=0.8$; one can obtain the parameters of fault detection filters and fault tolerant controllers by the aid of equations (46)-(48) in Theorem 2. Thus, the simulation results are shown in Figures 1-5. The trajectories of residual signal and residual evaluation function are shown in Figure 1. One can obtain that the residual can estimate the fault signal accurately. The threshold $J_{\text {th }}=0.0054$ and the detection time is $0.02 \mathrm{~s}$. Thus, the fault detection filters in this paper can detect the fault signal efficiently. The response of attack angle is depicted in Figure 2; we can conclude that the response of attack angle can track the input signal, even when there exist disturbance and fault signal. The fault signal will lead to performance degradation, but

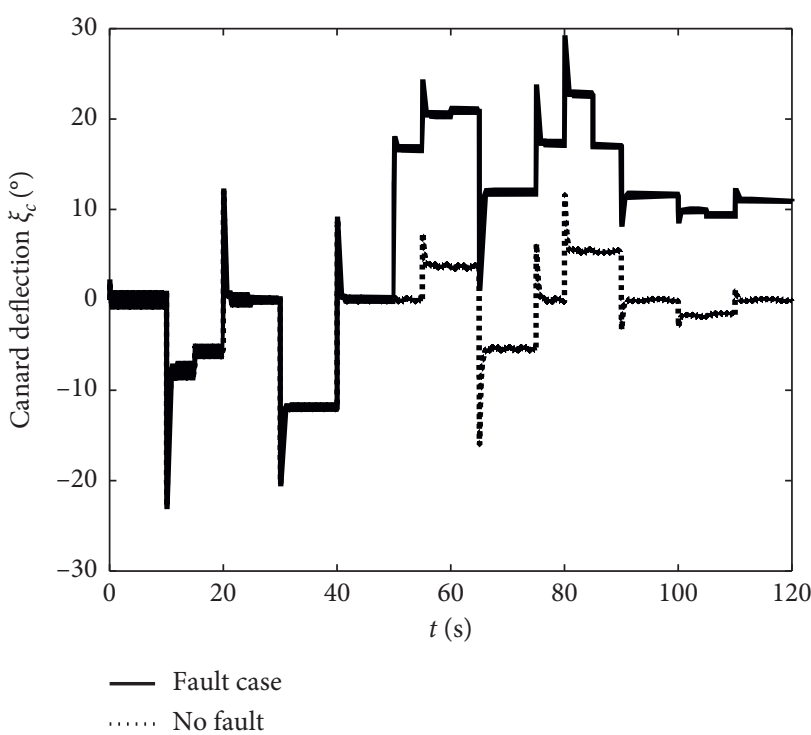

Figure 5: Response of canard deflection.

stability and tracking performance can be guaranteed by the aid of fault tolerant controller. Moreover, the control surface deflections are shown in Figures 3-5. We can see that they are practical and achievable. Based on the statement above, we can conclude that the fault detection filters can detect the fault signal effectively and the fault tolerant controller can ensure the stability and tracking performance.

In order to demonstrate the superiority of the proposed method, the comparisons are given in this section.

The MDADT method is introduced in this paper. Compared to the traditional ADT method, the properties of each subsystem are taken into consideration and less conservative results are obtained. We can obtain the results of the switched system under MDADT method and ADT method in Table 2, from which we can conclude that the 
TABle 2: Coefficients and results for the system under different switching schemes.

\begin{tabular}{|c|c|c|}
\hline $\begin{array}{l}\text { Switching } \\
\text { schemes }\end{array}$ & MDADT switching & ADT switching \\
\hline Coefficients & $\begin{array}{c}a_{1}=0.25, a_{2}=0.21, a_{4}=0.19, a_{8}=0.19, a_{9}=0.25, a_{12}=0.23, \\
a_{18}=0.24, a_{19}=0.25 \\
b_{1}=0.08, b_{2}=0.10, b_{4}=0.11, b_{8}=0.11, b_{9}=0.09, b_{12}=0.10, \\
\\
b_{18}=0.08, b_{19}=0.08 \\
\mu_{11}=1.03, \mu_{12}=1.05, \mu_{14}=1.06, \mu_{18}=1.03, \mu_{19}=1.02, \\
\mu_{112}=1.03, \mu_{118}=1.04, \mu_{119}=1.06 \\
\mu_{21}=1.11, \mu_{22}=1.15, \mu_{24}=1.18, \mu_{28}=1.11, \mu_{29}=1.09, \\
\mu_{212}=1.12, \mu_{218}=1.08, \mu_{219}=1.13 \\
\Delta_{1}=2, \Delta_{2}=3, \Delta_{4}=3, \Delta_{8}=1, \Delta_{9}=2, \Delta_{12}=1, \Delta_{18}=2, \Delta_{19}=2\end{array}$ & $\begin{array}{c}a_{1}=a_{2}=a_{4}=a_{8}=a_{9}=a_{12}=a_{18}=a_{19}=0.19 \\
b_{1}=b_{2}=b_{4}=b_{8}=b_{9}=b_{12}=b_{18}=b_{19}=0.11 \\
\mu_{11}=\mu_{12}=\mu_{14}=\mu_{18}=\mu_{19}=\mu_{112}=\mu_{118}=\mu_{119}=1.06 \\
\mu_{21}=\mu_{22}=\mu_{24}=\mu_{28}=\mu_{29}=\mu_{212}=\mu_{218}=\mu_{219}=1.18 \\
\Delta_{1}=\Delta_{2}=\Delta_{4}=\Delta_{8}=\Delta_{9}=\Delta_{12}=\Delta_{18}=\Delta_{19}=3\end{array}$ \\
\hline Results & $\begin{array}{c}\tau_{a 1}^{*}=7.7429, \tau_{a 2}^{*}=19.8517, \tau_{a 4}^{*}=33.8628, \tau_{a 8}^{*}=13.0060 \\
\tau_{a 9}^{*}=7.6576, \tau_{a 12}^{*}=5.8657, \tau_{a 18}^{*}=8.3366, \tau_{a 19}^{*}=8.1606\end{array}$ & \\
\hline
\end{tabular}

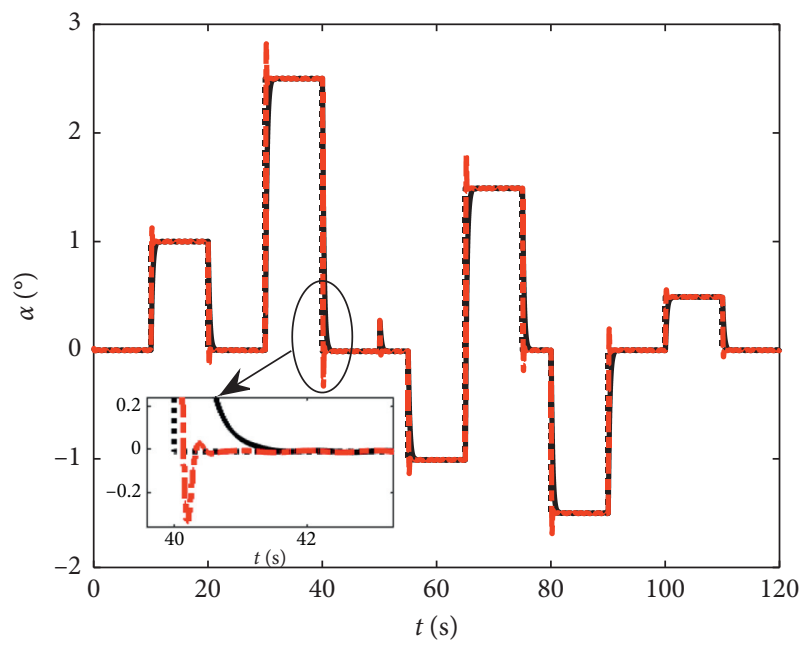

— Angle of attack

..... Commond signal

-. - Angle of attack_s

Figure 6: Response of attack angle.

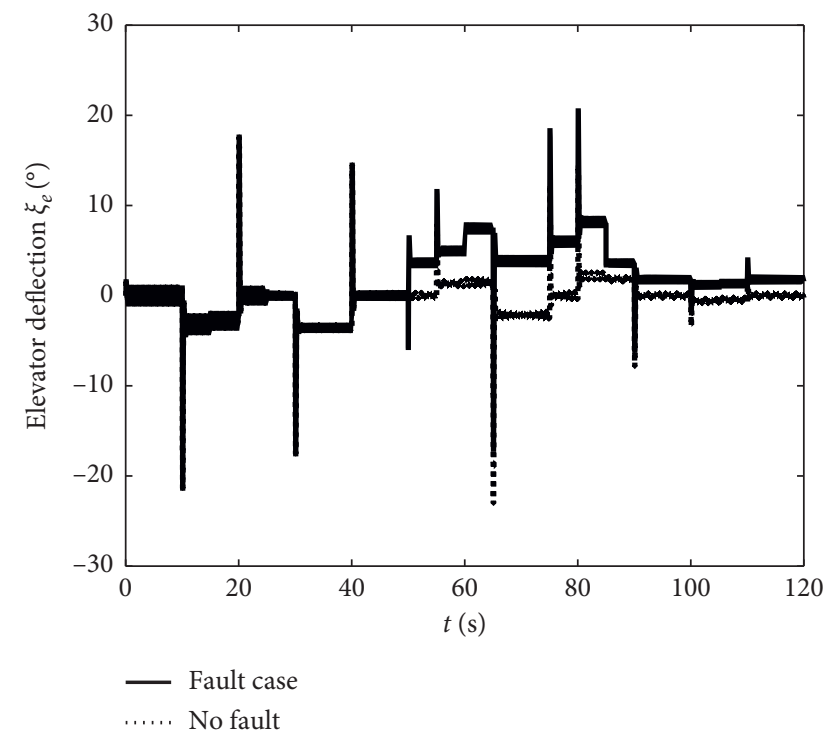

Figure 7: Response of elevator deflection.

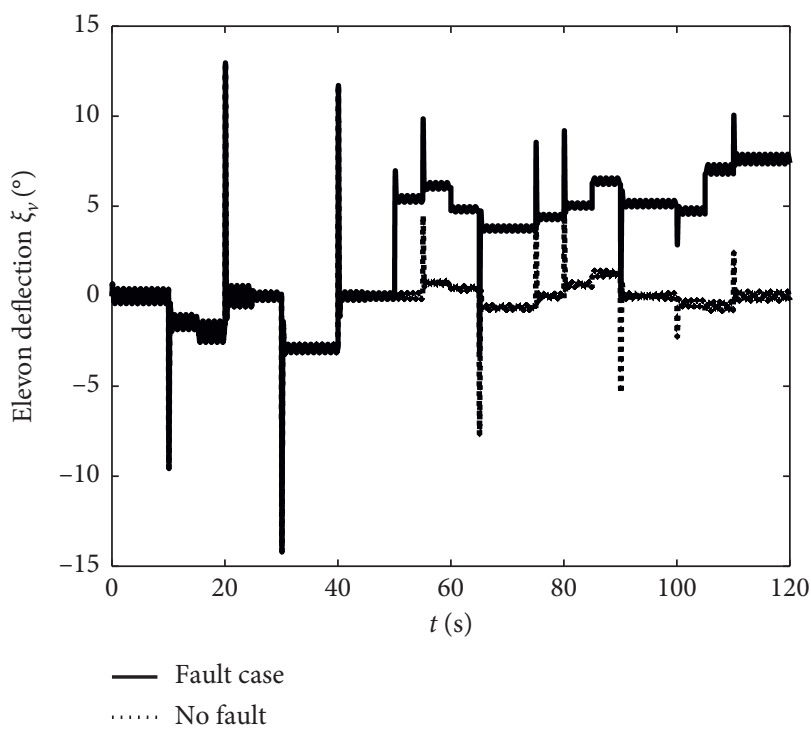

FIgURE 8: Response of elevon deflection.

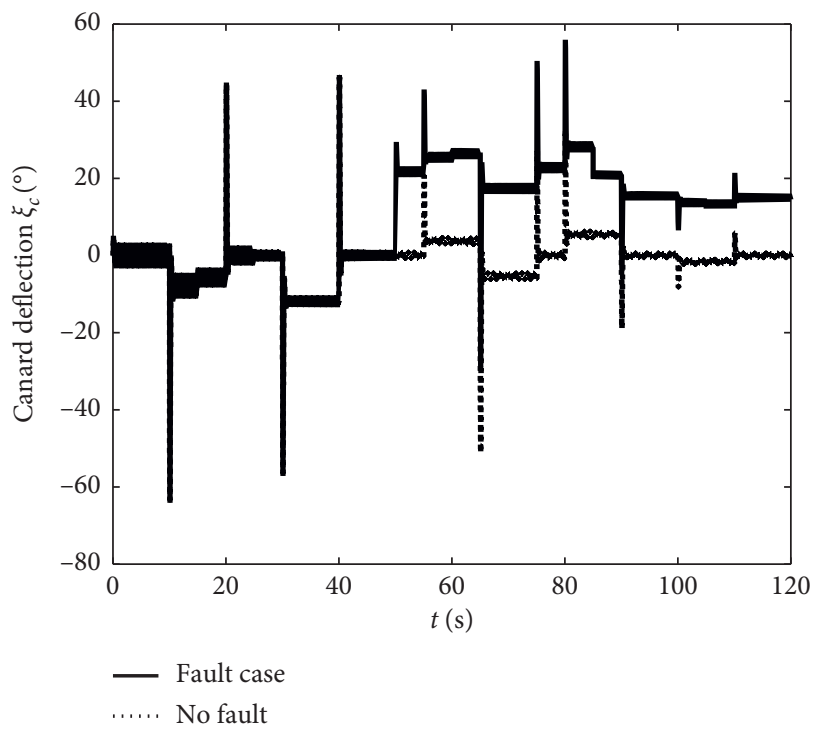

Figure 9: Response of canard deflection. 
MDADT method is less conservative and ADT method can be viewed as a special case of MDADT method.

In addition, the integrated design method can achieve optimal performance and reduce complexities; the results of separated design method via MDADT method are given in Figures 6-9. It is assumed that we design the fault tolerant controller firstly, and then the fault detection filter is introduced in the closed-loop system. The response of attack angle is shown in Figure 6; we can see that the fault detection filter will influence the performance of fault tolerant controller. The transient performance of integrated design method is better than that of separated design method. The responses of actuators are shown in Figures 7-9; we can conclude that the response of separated design method is achievable, but it will lead to performance degradation because of the fault detection filter.

\section{Conclusion}

The problem of integrated fault detection and fault tolerant control for switched systems with asynchronous switching is investigated in the paper. Considering the data missing from output to filter/controller, the model of switched with asynchronous switching is established. To achieve optimal performance, the output tracking controller and fault detection filter are designed simultaneously. The multiple Lyapunov functional method and MDADT are combined to guarantee the finite-time stability of the closed-loop system, which leads to less conservative results. The solutions of the filters and controllers are given in the form of LMIs. Simulation in the end demonstrates the effectiveness of the proposed method.

\section{Data Availability}

The simulation data used to support the findings of this study are included within the article.

\section{Conflicts of Interest}

The authors declare that they have no conflicts of interest.

\section{Acknowledgments}

This work was supported by Fundamental Research Funds for the Central Universities (G2018KY0301) and the National Natural Science Foundation of China (61603297).

\section{References}

[1] B.-L. Su, D. Dan, and Y. Chun, "Finite-time optimization stabilization for a class of constrained switched nonlinear systems," Mathematical Problems in Engineering, vol. 2018, Article ID 6824803, 8 pages, 2018.

[2] X. Li, R. K. Hamid, and Z. G. Xiang, "Robust reliable control of uncertain discrete impulsive switched systems with state delays," Mathematical Problems in Engineering, vol. 2013, Article ID 197819, 8 pages, 2013.

[3] X. Li, J. Cao, and M. Perc, "Switching laws design for stability of finite and infinite delayed switched systems with stable and unstable modes," IEEE Access, vol. 6, pp. 6677-6691, 2018.
[4] H. Cheng, C. Dong, W. Jiang, Q. Wang, and Y. Hou, "Nonfragile switched $H_{\infty}$ control for morphing aircraft with asynchronous switching," Chinese Journal of Aeronautics, vol. 30, no. 3, pp. 1127-1139, 2017.

[5] Z. Fang, X.-Z. Huang, and X.-G. Tan, "Stability of stochastic differential switching systems with time-delay and impulsive effects," Mathematical Problems in Engineering, vol. 2018, Article ID 3841675, 9 pages, 2018.

[6] D. Du, B. Jiang, and P. Shi, "Fault detection for discrete-time switched systems with intermittent measurements," International Journal of Control, vol. 85, no. 1, pp. 78-87, 2012.

[7] G.-L. Wang and H.-Y. Cai, "Disordered stabilization of stochastic delay systems: the disorder-dependent approach," Mathematical Problems in Engineering, vol. 2017, Article ID 7057628, 13 pages, 2017.

[8] J. Wang and J. Zhao, "Stabilisation of switched positive systems with actuator saturation," IET Control Theory \& Applications, vol. 10, no. 6, pp. 717-723, 2016.

[9] L. An, D. Zhai, J. Dong, and Q. Zhang, "LMI-based adaptive reliable $H_{\infty}$ static output feedback control against switched actuator failures," International Journal of Systems Science, vol. 48, no. 11, pp. 2345-2355, 2017.

[10] X. Zhao, Y. Yin, L. Zhang, and H. Yang, "Control of switched nonlinear systems via T-S fuzzy modeling," IEEE Transactions on Fuzzy Systems, vol. 24, no. 1, pp. 235-241, 2016.

[11] Y. Wang, N. Roohi, G. E. Dullerud, and M. Viswanathan, "Stability analysis of switched linear systems defined by regular languages," IEEE Transactions on Automatic Control, vol. 62, no. 5, pp. 2568-2575, 2017.

[12] Y. Wei, J. H. Park, J. Qiu, L. Wu, and H. Y. Jung, "Sliding mode control for semi-Markovian jump systems via output feedback," Automatica, vol. 81, pp. 133-141, 2017.

[13] P. Zhao and R. Nagamune, "Switching LPV controller design under uncertain scheduling parameters," Automatica, vol. 76, pp. 243-250, 2017.

[14] L. Zhang and H. Gao, "Asynchronously switched control of switched linear systems with average dwell time," Automatica, vol. 46, no. 5, pp. 953-958, 2010.

[15] X. Zhao, L. Zhang, P. Shi, and M. Liu, "Stability and stabilization of switched linear systems with mode-dependent average dwell time," IEEE Transactions on Automatic Control, vol. 57, no. 7, pp. 1809-1815, 2012.

[16] B. Niu, P. Zhao, J. D. Liu, H.-J. Ma, and Y.-J. Liu, "Global adaptive control of switched uncertain nonlinear systems: an improved MDADT method," Automatica, vol. 115, Article ID 108872, 2020.

[17] B. Niu, D. Wang, M. Liu, X. Song, H. Wang, and P. Duan, "Adaptive neural output-feedback controller design of switched nonlower triangular nonlinear systems with time delays," IEEE Transactions on Neural Networks and Learning Systems, pp. 1-10, 2019.

[18] B. Niu, Y. Liu, W. Zhou, H. Li, P. Duan, and J. Li, "Multiple Lyapunov functions for adaptive neural tracking control of switched nonlinear nonlower-triangular systems," IEEE Transactions on Cybernetics, vol. 50, no. 5, pp. 1877-1886, 2020.

[19] X. Zhao, P. Shi, Y. Yin, and S. K. Nguang, "New results on stability of slowly switched systems: a multiple discontinuous Lyapunov function approach," IEEE Transactions on Automatic Control, vol. 62, no. 7, pp. 3502-3509, 2017.

[20] D. S. Dong, S. Y. Xu, and C. Vincent, "Fault detection for nonlinear discrete-time switched systems with persistent dwell time," IEEE Transactions on Fuzzy Systems, vol. 26, no. 4, pp. 2466-2474, 2018. 
[21] S. Ifqir, I. Dalil, A.-O. Naima, and M. Said, "Adaptive threshold generation for vehicle fault detection using switched T-S interval observers," IEEE Transactions on Industrial Electronics, vol. 67, no. 6, pp. 5030-5040, 2020.

[22] H. Zhang, K. Zhang, Y. Cai, and J. Han, “Adaptive fuzzy faulttolerant tracking control for partially unknown systems with actuator faults via integral reinforcement learning method," IEEE Transactions on Fuzzy Systems, vol. 27, no. 10, pp. 1986-1998, 2019.

[23] S. Sun, H. Zhang, J. Zhang, and K. Zhang, "Multiple intermittent fault estimation and tolerant control for switched T-S fuzzy stochastic systems with multiple time-varying delays," Applied Mathematics and Computation, vol. 377, Article ID 125114, 2020.

[24] J. Han, X. Liu, X. Wei, X. Hu, and H. Zhang, "Reduced-order observer based fault estimation and fault-tolerant control for switched stochastic systems with actuator and sensor faults," ISA Transactions, vol. 88, pp. 91-101, 2019.

[25] Z. Wang, Q. Wang, L. Gong, and C. Gong, "Closed-loop fault detection for full-envelope flight vehicle with measurement delays," Chinese Journal of Aeronautics, vol. 28, no. 3, pp. 832-844, 2015.

[26] H. Cheng, W. Fu, C. Dong, Q. Wang, and Y. Hou, "Asynchronously finite-time $H_{\infty}$ control for morphing aircraft," Transactions of the Institute of Measurement and Control, vol. 40, no. 16, pp. 4330-4344, 2018.

[27] G. L. Hartmann, M. F. Barrett, and C. S. Greene, Control Design for an Unstable Vehicle,NASA, Washington, DC, USA, 1979. 